\title{
Fat Grafting for Maximizing the Volume of Breast Reconstruction by Latissmus Dorsi Mini-Flap
}

A.A.Salem, G.S.Saleh, A.M.Nawar, M.E.Abdellatif and A.G.Mohamed

General Surgery Dept., Faculty of Medicine, Benha Univ., Benha, Egypt

E-Mail:amr.g.m.2010@gmail.com

\begin{abstract}
The oncoplastic surgery (OPS) technique, which was first attempted by Audretsch et al., expands on the concept of breast-conserving surgery[1]. The pedicled latissimus dorsi (LD) flap serves an important function in breast reconstruction, but its utility is limited by its inability to provide sufficient volume [2]. Autologous expansion of the LD fold with lipomove has been utilized to keep away from situation of an embed. We improve the method by performing lipo-move during bosom recreation [3]. The examination was a planned, relative investigation incorporates 30 female patients with bosom malignancy stages, introduced to Benha University Hospital during the period from March 2018 to May 2020.All patients gave bosom knots, both ways sided, the ages at time of introduction went from 36 to 57 years (normal 46 years).they were partitioned into two gatherings as indicated by sort of offered substitution strategy: Group (A): by utilizing smaller than expected latissimus dorsi muscle fold just and Group (B): by utilizing scaled down latissimus dorsi muscle fold notwithstanding quick intraoperative fat joining. As of late, fat uniting has been demonstrated to be a promising restorative methodology for scar the executives after consumes and physical injury. This method can be utilized in the setting of a LD fold/tissue expander/embed recreation, and could likewise be extended to different autologous bosom reproduction choices [4].
\end{abstract}

Keywords: OPS, Reconstructive Breast Surgery, Latissimus Dorsi Flap \& Fat Grafting.

\section{Introduction}

The expanding frequency of bosom malignant growth has made it one of the most pervasive tumors in ladies around the world. With the ongoing progressions in symptomatic regimens for bosom malignant growth and the universalized utilization of normal registration, little, beginning time bosom diseases are progressively being recognized. Along these lines, bosom preserving medical procedure is much of the time performed, and endeavors have been made to create methods to limit postoperative bosom distortion after incomplete mastectomy [5].

Oncoplastic medical procedure offers a superior corrective result as fractional bosom remaking, utilizing different methods, when performed during a similar technique. In fractional bosom reproduction, remedial mammaplasty strategies offer imaginative choices for enormous and pendulous bosom. Then again, perforator folds, which save latissimus dorsi muscle work, give significant technique to little estimate bosoms [6].

The pedicled latissimus dorsi (LD) fold serves a significant capacity in bosom reproduction, yet its utility is restricted by its powerlessness to give adequate volume [7].

Another methodology is the all-inclusive LD fold procedure, which includes progressively forceful collect of subcutaneous tissue alongside the skin paddle. The deficiencies of this strategy incorporate a higher danger of seroma, wound breakdown, form deformations, and lumbar hernia [8].

Absolutely autologous bosom remaking joining LD smaller than expected fold and intra-pectoral fat uniting in a similar methodology is another procedure permitting expanded bosom volume in a solitary medical procedure [9].

\section{Patient \& methods}

The investigation was a planned, near examination incorporates 30 female patients with bosom malignant growth stages, introduced to Benha University Hospital during the period from March 2018 to May 2020.

An aggregate of 30 female patients with malignancy bosom were designated to experience distinctive mastectomy methods as indicated by the site of the tumor and the extraction volume corresponding to the bosom size with prompt recreation by smaller than normal Latismus-Dorsi muscle fold just as opposed to including intraoperative fat joining $+/$ - another extra fat infusion sits.

All patients gave bosom bumps, both ways sided, the ages at time of introduction went from 36 to 57 years (normal 46 years).

\section{Incorporation models}

Age beneath 60 years of age.

Resection of over $40 \%$ of the bosom volume.

Central, horizontal, lower and upper quadrants tumors.

Axillary analyzation through independent cut away from lumpectomy entry point.

Incomplete preparation of bosom parenchyma to permit reshaping of the bosom.

Women considering a bosom reproduction notwithstanding extraction.

\section{Prohibition models}

When clear edges can't be guaranteed without performing mastectomy.

Patients with T4 tumors.

Those patients with multicentric infection.

Patients with broad harmful mammographic miniaturized scale calcification.

Patients with incendiary carcinoma.

Patients with history of past radiation to the chest or axilla and axillary dismemberment.

Patients who have experienced an extreme neck dismemberment with penance of the spinal frill nerve. 
Patients with stomach hernia of past major exploratory stomach activities for Group B patients' determination.

In patients with uncontrolled co-morbidities (diabetes-chest issues......and so on)

The members who consented to partake in this clinical investigation gave educated assent subsequent to being completely educated about the strategy and its conditions.

\section{Patients' counseling}

Patients were by and large directed that the medical procedure would take 4 to 5 hours for a run of the mill latissimus dorsi fold bosom remaking. Postoperatively, they would have 2 contributor site channels and 1 to 2 bosom channels, which would stay until yields were underneath $30 \mathrm{~mL} / \mathrm{d}$, or by and large up to 3 or a month.

By and large, patients would stay in the clinic for 1-4 days. They may begin furthest point and scope of movement practices fourteen days after medical procedure progressively and could envision recovering ordinary capacity for exercises, for example, driving and coming back to work in 3 to about a month and a half.

\section{Patients' gathering}

In our examination the 30 patients who finished a follow up for 10 months were remembered for this investigation and were partitioned into 2 gatherings.

\section{The two gatherings were treated by}

Wide neighborhood extraction of the bosom mass with satisfactory wellbeing edges under the umbrella of intraoperative solidified area assessment.

Axilla was treated by either sentinel lymph hub approach or all out axillary leeway approach through a different entry point.

Breast remaking was offered by small scale Latissimus Dorsi muscle fold with or without skin paddle.

Gathering A: This gathering of (15) patients was remade with small scale Latissimus Dorsi muscle fold alone.

Gathering B: This gathering of (15) patients was remade with smaller than usual Latissimus Dorsi muscle fold with quick intra-employable fat joining.

In the two gatherings, every one of the (30) patients were treated by bosom mass extraction agreeing:

Preoperative readiness and drawings:

The objective of ideal usable method is to augment the delicate tissue inclusion gave by the fold, while limiting the greatness of benefactor site deformity and contributor site difficulties.

Markings are performed preoperatively with the patient in the upstanding position and anteriorly incorporate the midline, inframammary crease and sidelong edge of bosom tissue and posteriorly incorporate horizontal edge of the latissimus along the back axillary line, unrivaled edge at the tip of the scapula, and second rate edge at the iliac peak.

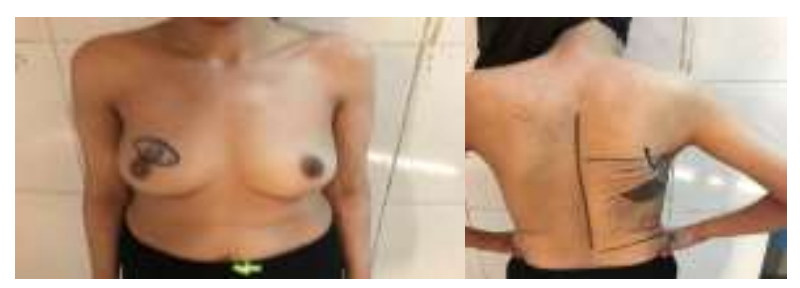

Fig (1) Pre-operative drawngs

In both groups, axillary lymph nodes were treated in all (30) patients eiher by

Sentinel lymph node biopsy: which was done for only 6 patients

A harmless blue dye was injected into the breast: under or around the areola, which is the darker area that surrounds the nipple. Then, an incision was done under the arm at the axillary line to find the lymph nodes that were stained from the dye and removed. A pathologist carefully examined the sentinel node(s) for signs of cancer.

\section{Axillary lymph node dissection: which was done for} (24) patients

While awaiting the results of frozen section, the site of incision for axillary access is marked below the axillary line providing a very wide access to the axilla which greatly facilitate level I and level II lymph node dissection which was carried in all patients without excessive traction for exposure or injury to the significant structures in the axilla.

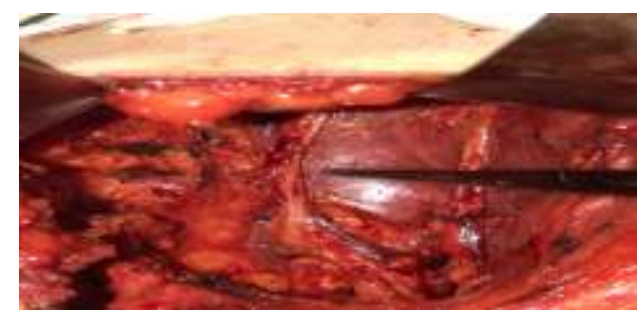

Fig (2) Dissection over and preservation of the thoracodorsal bundle.

In the two gatherings (30) patients, bosom surrenders were reproduced by smaller than usual Latissimus dorsi muscle fold

In the working room, the patient is put in the horizontal decubitus position for one-sided or inclined situation for two-sided fold height. Dismemberment is done underneath the thoracolumbar sash, leaving the profound fat appended to the back skin folds. The latissimus is isolated from the serratus front at the parallel fringe: from the para-spinous muscle sash, lumbosacral belt, and vertebral section: from the trapezius strands superomedially: and from the teres significant filaments in the axilla.

After distinguishing proof of the thoracodorsal vessels, the latissimus is isolated close to its connection to the humerus. The myocutaneous or myofascial fold is then moved to the mastectomy deformity through a 
subcutaneous passage in the axilla. The patient is then positioned in the prostrate position, and the specialist continues with fold situation.

At last, addition of a couple of vacuum depletes, the injury is shut in two layers utilizing vycril (polygalactine) sutures for both subcutaneous and subcuticular layers. No deep sutures were used for all wounds.
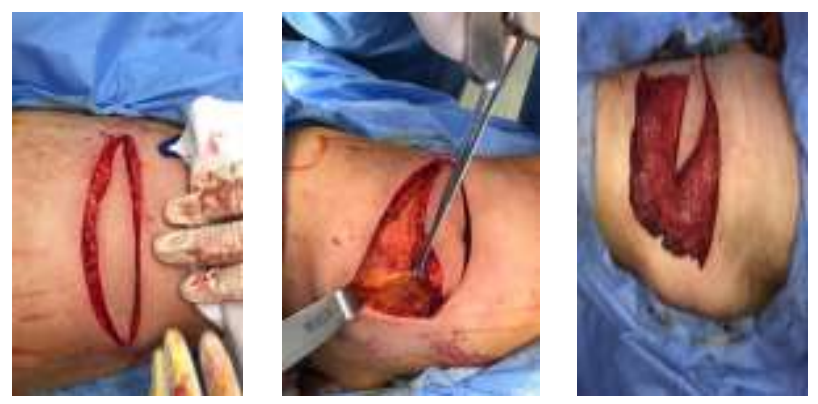

Fig (3) Dissection of mini-LD flap

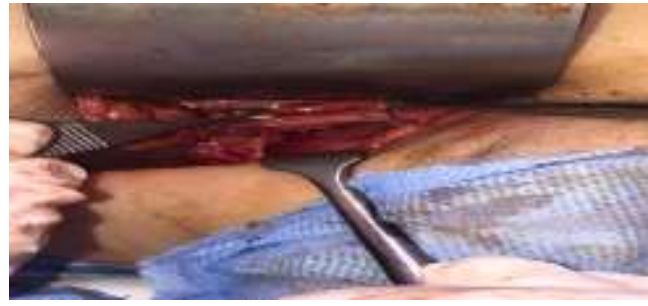

Fig (4) Division of the thoracodorsal nerve to the LD muscle after complete localization of the muscular flap in the breast defect.

Group B (15) patients: Breast reconstruction was super added by immediate intraoperative fat grafting.

\section{Fat harvesting}

Fat gather was performed all the while with the wide nearby extraction and LD fold reap, using a two-group approach, so as to limit the activity time or in the wake of completing the fold collect in pool of 2 group approach, yet in longer time (around 30-50 minutes).

The entry points were performed with a no. 15 surgical tool, and their position relies upon the region to be reaped.

The stomach fat is reaped through periumbilical or supra-iliac cuts.

The flanks through supra-iliac cuts, one on each side.

The "wet" procedure was utilized by invasion of a distended liquid for example Klein arrangement containing a nearby sedative medication $(10 \mathrm{cc}$ lignocaine) and $0.25-1 \mathrm{mg}$ epinephrine, in $500 \mathrm{cc}$ Ringer's answer or Normal Saline.

Coleman procedure for fat was utilized with a 3-mm, obtuse edged, 2-opening cannula associated with a 10$\mathrm{mL}$ syringe: fat is suctioned physically by pulling back the unclogger.
A liposuction of around $700 \mathrm{ml}$ all out suction (run: 250-2500 ml) was performed by and large. Giver locales shifted with an attention on the mid-region and the flanks.

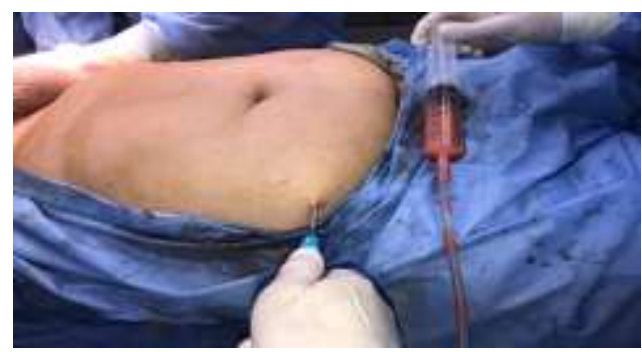

Fig (5) Manual liposuction technique: two team approach as liposuction was done simultaneous with breast mass excision.

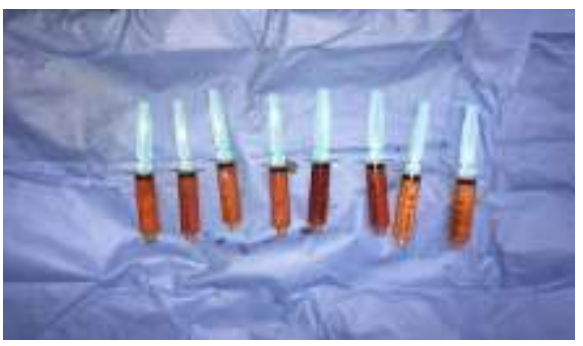

Fig (6) lipoaspirate collection in syringes before fat processing

\section{Fat processing}

Once the fat is harvested, it was centrifuged at 3000 rpm for $3 \mathrm{~min}$. After the centrifugation, three layers were observed:

The first (upper) oily layer of lipids, which leaked out of disrupted adipocytes and could be poured off using absorbent material.

The second (middle) layer consisted of purified fatty tissue. This was routinely used for fat grafting.

The third (lower) layer contained blood, debris, tissue fluid, and local anesthetic and was ejected from the base of syringe.

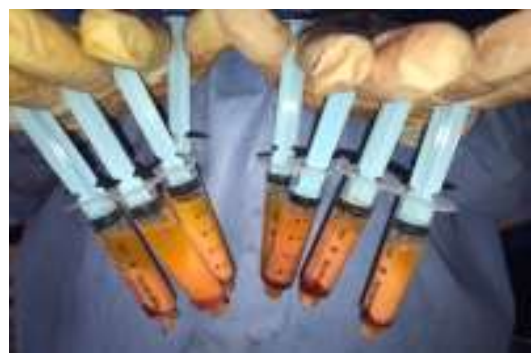

Fig (7) Centrifuged fat syringes after processing showing the 3 different layers.

To separate the hematic level, the bottom plug was opened and the blood was allowed to flow out onto a tray. The broken down fatty acids in the top level could be removed by decantation and if necessary the oil could be removed with the aid of small lined gauze. 


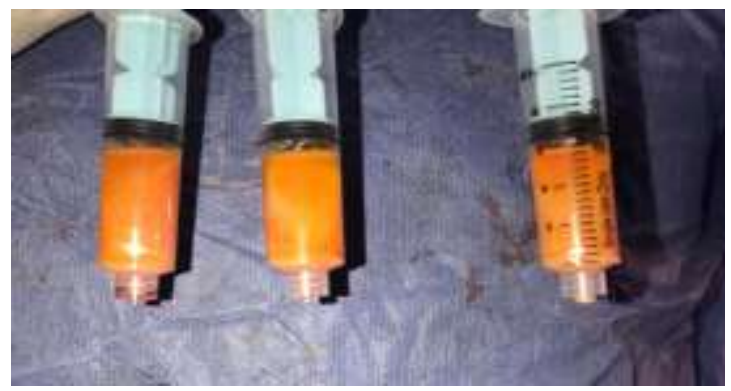

Fig (8) Fat syringes after filtration and getting rid of the hematic layer: which are ready for fat injection.

After the filtration process, an average volume of 220 $\mathrm{ml}$ (range: $70-450 \mathrm{ml}$ ) was available for lipo-filling. This is equivalent to $20 \%$ of the initially harvested volume.

\section{Fat grafting}

The skin of the breast was punctured with an 18gauge cannula that was used to release dermato-fascial adhesions and scar tissue. The same cannula was then used to inject the fat graft in the subcutaneous and subglandular plane of the breast.

Usually, through multiple access sites, multiple tunnels were created on insertion, but fat was injected only during withdrawal of the cannula in a "fanning-out" pattern, to avoid excessive interstitial pressure at the recipient site and overcrowding of the transplanted adipocytes following the fat "spaghetti" principle.

The mastectomy skin flaps as well as the pectoralis major muscle were fat grafted before the inset of the LD flap

For the LD flap, fat was grafted into the subcutaneous layer of the skin paddle, the LD muscle caudal to the skin paddle, and under the muscle fascia.

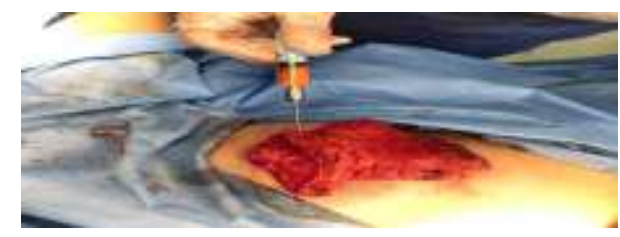

Fig (9) Fat grafting step in the subcutaneous layer of the skin paddle after its de-epithelialization

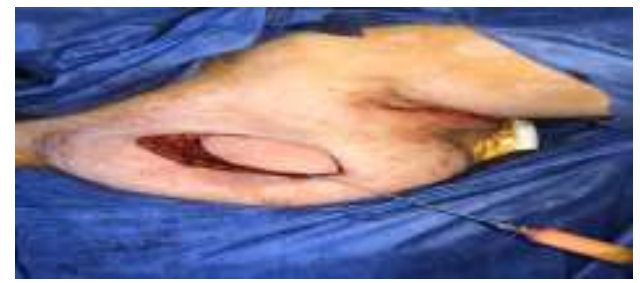

Fig (10) Fat grafting step in the subcutaneous layer of the skin paddle

As required, additional fat grafting was performed 4 to 10 months following the initial breast reconstruction procedure. The fat grafting injections focused on correcting upper pole efficiency and increasing the projection of the reconstructed breast.

\section{Results}

This investigation is imminent investigation done in Benha University including thirty female patients were followed up for ten months: every one of them experienced total bosom assessment and bosom multidisciplinary group appraisal for offering the best treatment plan for every individual patient. All patients experienced bosom traditionalist mastectomy of wide nearby extraction and axillary lymph hubs evaluation (either sentinel biopsy or complete analyzation) at that point they were separated into two gatherings as per kind of offered substitution method:

Gathering (A): by utilizing smaller than usual latissimus dorsi muscle fold as it were

Gathering (B): by utilizing small latissimus dorsi muscle fold notwithstanding quick intraoperative fat joining.

Individual information, grumbling and history

The age was gone from 36 to 57 years (with normal 46 years). All patients were given a bosom protuberance and none of them was given some other manifestations. Family ancestry of bosom disease in the first or the second degree family members was sure in just 6 patients (20\%). 21 patients were premenopausal $(70 \%)$ while 9 were postmenopausal $(30 \%)$. All patients were multiparous aside from 3 of them were nulliparous $(10 \%)$. Six patients $(20 \%)$ had controlled hypertension. Different comorbidities experienced were bronchial asthma in one patient and gentle mitral stenosis infection in another patient in which the conditions were controlled medicinally. Twenty-one patients (70\%) had gotten preoperative chemotherapy as neoadjuvant treatment for persistent down arranging.

Seventeen patients $(56.7 \%)$ were hefty (Body Mass Index surpassing $30 \mathrm{~kg} / \mathrm{m} 2$ ): none had proof of general heath weakness, metastatic malady or pregnancy. Eighteen patients had left bosom malignant growth $(60 \%)$ while twelve had right bosom disease $(40 \%)$ and none had reciprocal love.

\section{Sort of Cancer}

Last histopathology uncovered the conclusion of IDC in twenty-one patients (70\%). nine patients $(30 \%)$ had the conclusion of ILC.

\section{a. Tumor Molecular order}

We found that twelve patients (40\%) were named Luminal A, nine patients (30\%) were named Luminal B, while six patients (20\%) were delegated Basal likeHer2neo and three patients (10\%) were named triple negative.

\section{b. Lymhatic Involvement}

At conclusive histopathological report, three patients $(10 \%)$ had lymph hubs liberated from tumor association. Eighteen patients had N1 positive lymph hubs $(60 \%)$ and nine patients $(30 \%)$ had N2 positive lymph hubs. 


\section{c. Neoadjuvant and Adjuvant Therapy}

Twenty one patients $(70 \%)$ had gotten preoperative neoadjuvant chemotherapy for quiet down-organizing as per the common choice with our bosom MDT group.

All patients $(100 \%)$ had postoperative radiotherapy. Eighteen patients $(60 \%)$ had support on the past tumor site after intraoperative cut-out.

Chemotherapy (FAC or FEC 6 cycles) was given to 27 patients $(90 \%)$ who finished their cycles before the beginning of radiotherapy. Twenty one patients $(70 \%)$ got hormonal treatment: two of them ended hormonal treatment following one year because of awful consistence and symptoms. The other nine patients (30 $\%$ ) had negative hormonal receptor status and hormonal treatment was not given.

\section{Postoperative development}

Gathering A (Reconstruction by scaled down LD solid fold just): 15 cases

- The usable time extended between 270-400 minutes with mean usable time of 330.5 minutes including the time taken for prompt histopathological assessment of the examples evacuated for solidified segment assessment.

- The medical clinic stay interim was extended between 1-3 days with normal 2 days postoperative remain, in which wound and fold assessment was done cautiously.

- Immediate postoperative before release:

1. Acceptable tissue edema in the beneficiary site.

2. Minimal to gentle liquid in the channel attractions framework.

3. Three patients (20\%) had fold clog and skin ecchymosis. All patients had good taken flap with no evidence on flap ischemia or necrosis.

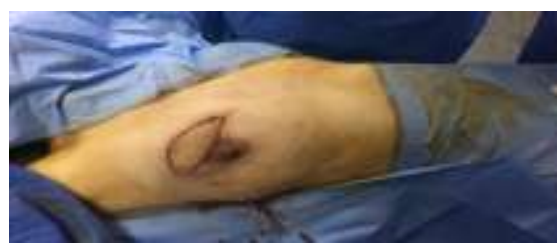

Fig (11) Immediate post-operative with suction drains in the back and axilla.

\section{- During the first visit (after 1 week)}

1. Two patients (13.3\%) had superficial surgical site infection at the donor site managed conservatively by systemic antibiotics and frequent dressings.

2. One patient $(6.7 \%)$ had superficial surgical site infection at the recipient site due to bad personal hygiene, managed conservatively by systemic antibiotics and frequent dressings.

3. Four patients $(26.7 \%)$ had developed seroma at the donor site in the back and aspirated radiologically guided.

4. One patient $(6.7 \%)$ had developed hematoma at the donor site in the back and managed conservatively.
5. None of the patients had wound dehiscence neither at donor nor recipient sites.

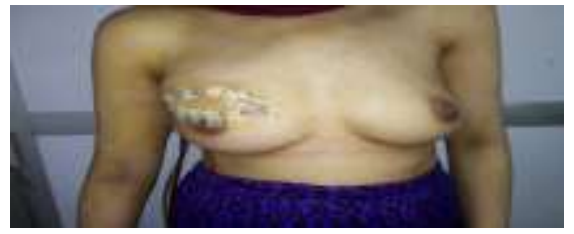

Fig (12) One week post-operative with suction drains in the back and axilla.

\section{- During the second visit (after 1 month)}

All patients who had shallow careful site contamination at the contributor and beneficiary locales were recuperated totally.

All patients who had created seroma or hematoma at the giver site in the back had been soothed totally with evacuation all things considered.

Two patients (13.3\%) had created scar hypertrophy for the most part at contributor site in the back.

All patients were prepared even mentally for beginning the adjuvant treatment: radiotherapy, chemotherapy or hormonal treatment.

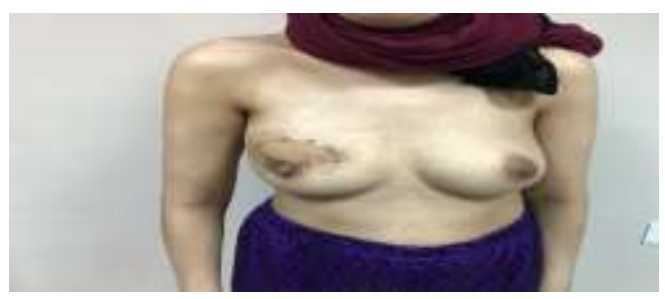

Fig (13) Multi month post-usable after evacuation of the attractions channels.

\section{During the third visit (following 3 months)}

Si patients $(40 \%)$ had mellow asymmetry between the two bosoms.

Nine patients $(60 \%)$ had mellow trouble with their day by day exercise and shoulder development.

Five patients $(33.3 \%)$ were introduced by early radiotherapy reactions as skin redness and disturbance.

\section{During the fourth visit (following a half year)}

Four patients (26.7\%) despite everything had mellow asymmetry between the two bosoms.

Six patients (40\%) despite everything had mellow trouble with their day by day exercise and shoulder development.

Three patients $(20 \%)$ were introduced by late radiotherapy symptoms as skin pigmentations.

One patient $(6.7 \%)$ had scar wretchedness at contributor site in the back.

1. Seven patients $(46.7 \%)$ were generally satisfied with the reconstruction results: others were unsatisfied about asymmetry, scar depression, reconstructed volume gradual shrinkage and difficulty in daily exercise. 


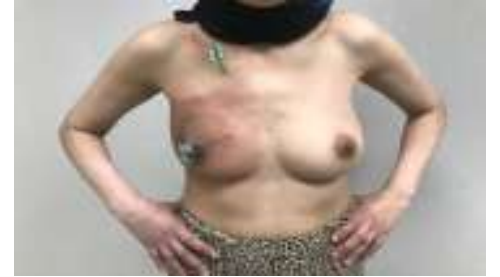

Fig (14) Three months post-operative with skin redness during radiotherapy sessions

\section{During the fifth visit (after 10 months)}

1. Three patients (20\%) still had mild asymmetry between the two breasts.

2. Three patients $(20 \%)$ still had mild difficulty with their daily exercise and shoulder movement.

3. Two patients (13.3\%) were presented by late radiotherapy side effects as reconstructed breast shrinkage.

4. Ten patients $(66.7 \%)$ were generally satisfied with the reconstruction results: others were unsatisfied about asymmetry, scar depression, reconstructed volume gradual shrinkage and difficulty in daily exercise.

Group B (Reconstruction by mini-LD muscular flap with immediate fat grafting): 15 cases

- The operative time ranged between 330-450 minutes with mean operative time of 405 minutes including the time taken for immediate histopathological examination of the specimens removed for frozen section examination, fat harvesting and grafting.

- The hospital stay interval was ranged between 2- 4 days with average 3 days postoperative stay, in which wound and flap examination was done carefully.

- Immediate postoperative before discharge:

1. Acceptable tissue edema in the recipient site.

2. Minimal to mild fluid in the drain suction system.

3. Minimal fluid discharge drained directly from the liposuction incision.

4. Two patients $(13.3 \%)$ had flap congestion and skin ecchymosis.

5. All patients had good taken flap with no evidence on flap ischemia or necrosis.

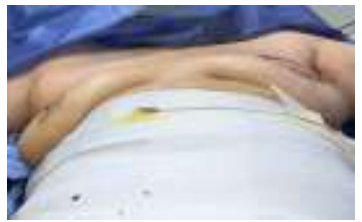

Fig (15) Immediate post-operative after fat grafting.

\section{- During the first visit (after 1 week)}

1. Two patients (13.3\%) had superficial surgical site infection at the donor site managed conservatively by systemic antibiotics and frequent dressings.

2. One patient $(6.7 \%)$ had abdominal subcutaneous ecchymosis.
3. Three patients $(20 \%)$ had developed seroma at the donor site in the back and aspirated radiologically guided.

4. Two patients $(13.3 \%)$ had developed hematoma at the donor site in the back and managed conservatively.

5. None of the patients had wound dehiscence neither at donor nor recipient sites.

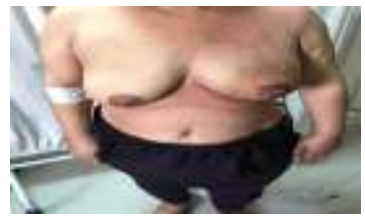

Fig (16) 1 week post-operative with suction drains

- During the second visit (after 1 month)

1. All patients who had superficial surgical site infection at the donor and recipient sites were recovered completely.

2. All patients who had developed seroma or hematoma at the donor site in the back had been relieved completely with removal of all drains.

3. The patient with abdominal ecchymosis had been recovered completely under conservative measurement.

4. One patient $(6.7 \%)$ had developed scar hypertrophy mainly at donor site in the back.

5. All patients were ready even psychologically for starting the adjuvant therapy: radiotherapy, chemotherapy or hormonal therapy.

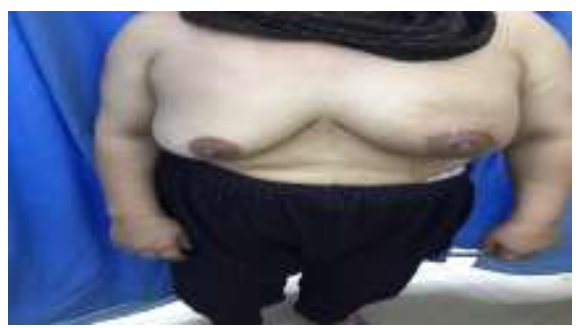

Fig (17) One month post-operative after removal of the suction drains in the back and axilla.

- During the third visit (after 3 months):

1. Two patients (13.3\%) had mild asymmetry between the two breasts: two of them were managed by additional fat grafting sits.

2. Nine patients $(60 \%)$ had mild difficulty with their daily exercise and shoulder movement.

3. Four patients $(26.7 \%)$ were presented by early radiotherapy side effects as skin redness and irritation.

\section{During the fourth visit (after 6 months)}

4. One patient $(6.7 \%)$ still had mild asymmetry between the two breasts and managed by additional fat grafting sits. 
5. Seven patients $(46.7 \%)$ still had mild difficulty with their daily exercise and shoulder movement.

6 . Three patients $(20 \%)$ were presented by late radiotherapy side effects as skin pigmentations.

7. Two patients $(13.3 \%)$ had scar depression at donor site in the back and were managed by another fat grafting sits.

8. Eleven patients $(73.3 \%)$ were generally satisfied with the reconstruction results: others were unsatisfied about asymmetry, scar depression, reconstructed volume gradual shrinkage and difficulty in daily exercise.

\section{During the fifth visit (after 10 months)}

9. Almost all patients had a symmetrical breasts after all additional fat grafting sits..

10. Two patients (13.3\%) still had mild difficulty with their daily exercise and shoulder movement.

11. Two patients (13.3\%) were presented by late radiotherapy side effects as reconstructed breast shrinkage and managed by additional fat grafting sits.

12. Fourteen patients $(93.3 \%)$ were generally satisfied with the reconstruction results, unsatisfaction about asymmetry, scar depression and reconstructed volume gradual shrinkage were managed by additional fat grafting sits.

\begin{tabular}{|c|c|c|}
\hline $\begin{array}{l}\text { Group (A) } \\
15 \text { patients under } \\
\text { went breast } \\
\text { reconstruction by } \\
\text { mini-latissimus }\end{array}$ & \multicolumn{2}{|c|}{$\begin{array}{l}\text { Group (B) } \\
15 \text { patients under } \\
\text { went breast } \\
\text { reconstruction by } \\
\text { mini-latissimus }\end{array}$} \\
\hline $330 \mathrm{~min}$ & Operation time & $405 / \min$ \\
\hline $80 \%$ & Symmetry & $\begin{array}{c}100 \% \\
\text { after additional } \\
\text { fat grafting } \\
\text { sessions }\end{array}$ \\
\hline $20 \%$ & Daily exercise difficulty & $20 \%$ \\
\hline $66.7 \%$ & Overall satisfaction & $93.3 \%$ \\
\hline
\end{tabular}

\section{Statistical analysis}

The collected data were summarized in terms of mean \pm Standard Deviation (SD) and range for quantitative data and frequency and percentage for qualitative data.

\section{Discussion}

A move in the worldview for bosom malignancy the board started in the late 1970s, when pioneers showed that the general endurance rates accomplished with mastectomy could be approached with bosom rationing tumor extraction and radiotherapy [10].

Bosom protection treatment with lumpectomy is a significant part of bosom malignant growth treatment, with a proportional endurance result to that of mastectomy [11,12]. Notwithstanding physical protection, ladies who experience bosom preservation have a superior perspective on their self-perception, are increasingly $\mathrm{OK}$ with bareness and bosom stroking, and may have less antagonistic physical sequelae from asymmetry, chest divider bonds and deadness related with mastectomy $[13,14]$.

Notwithstanding being proportional to mastectomy regarding oncological wellbeing, BCT offers points of interest over mastectomy as far as personal satisfaction and tasteful results. Personal satisfaction after bosom medical procedure is influenced by safeguarding of the bosom, dodging contributor site dismalness, restorative outcomes, treatment term, and accommodation. These elements must be considered in the appraisal of a patient who gives off an impression of being a fitting contender for BCT [15].

Oncoplastic bosom medical procedure (OPS) is generally new: right off the bat, oncoplastic strategies are justified to permit wide extraction and clear edges without bargaining cosmoses. Also, such medical procedure is undeniably performed simultaneously as oncological extraction [16].

Since Tansini [17] presented the method in 1896, using the LD fold has introduced a flexible choice for bosom remaking. Be that as it may, with the expanding prominence of using stomach based perforator folds, the LD fold has gotten the auxiliary decision for autologous bosom reproduction. The LD fold alone can't generally give adequate volume to the reproduced bosom [18].

Two great techniques have been applied for LD fold volume upgrade: embed inclusion and broadened LD fold gather. Embed addition is related with potential confusions, for example, contamination, unnatural bosom form, capsular constriction, embed relocation, and embed expulsion [19].

Expanded LD fold gathering can give extra volume without embeddings an embed, yet forceful tissue collecting may prompt expanded dangers of seroma, wound dehiscence, and form disfigurement. What's more, this procedure is confined to patients with adequate tissue at the fold giver site, which is normally connected with a higher BMI [20].

From March 2018 to May 2020: we began the assessment and correlation of having prompt fat uniting method with bosom remaking by smaller than usual Latissimus dorsi muscle fold versus the strong fold just for getting substantially more reproduction volume without greater dreariness to arrive at higher fulfillment. Prior to medical procedure, we followed the shred choice strategy by having a total clarification of the idea of the method, its advantages, dangers and potential difficulties were acquainted with all the patients.

Fat joining offers a less obtrusive technique for volume improvement. Since 2009 inversion of the American Society of Plastic Surgeons' ban on fat uniting, look into here has expanded dramatically. Several investigations have demonstrated that fat joining can fundamentally improve bosom shape and give extra volume in both autologous and embed based recreations and is likewise connected with moderately scarcely any intricacies, for example, disease and fat necrosis [21]. 
In 2010, Sinna et al [22] depicted the biggest case arrangement of second-stage fat joining in LD fold autologous bosom reproduction, which included 200 patients and 244 infusion meetings. In that review, the mean volume of fat joined per bosom was $176 \mathrm{~mL}$ (go, $30-405 \mathrm{~mL}$ ), and inconveniences included minor neighborhood disease $(0.8 \%)$ and fat putrefaction $(2.0 \%)$.The creator didn't obviously state in which layer the fat was infused, on the grounds that all the fat uniting revisional methods remembered for the investigation were performed by means of "daze" infusion. The assortment of writing on synchronous fat joining and autologous tissue bosom reproduction is exceptionally restricted, with just a solitary ongoing report of prompt fat uniting increased LD fold bosom reconstruction [23].

In this article, fat was infused into the fat layer and muscle belt of the fold skin paddle for volume upgrade of the lower shaft, with a mean infused fat volume of $220 \mathrm{~mL}$ (go, 70-450 mL).

As far as anyone is concerned, this investigation means to portray the prompt, multisite, and multilayer fat joining strategy for bosom recreation. Synchronous fat uniting into the LD fold was acted in all the cases in this arrangement, and no fat joining related inconveniences, fat embolism, or traded off LD fold vascularity because of this strategy were seen in this accomplice. The normal and maximal fat uniting volume for this situation arrangement was $220 \mathrm{~mL}$ and $450 \mathrm{~mL}$, separately,

Fat uniting can securely be performed into the subcutaneous layer of the LD skin paddle, under muscle sash, and inside the strong filaments caudal to the skin paddle during quick bosom reproductions, simultaneously as a LD fold move. Playing out the fatunited, volume-upgraded LD fold methodology with fat joining beneficiary locales can fill in as a helpful option in contrast to using midsection based folds in autologous bosom recreation, particularly to rescue a bombed embed or to play out a LD fold bosom reproduction [24].

This is a simple method for performing autologous bosom recreation in patients with a wide assortment of BMIs (go, 20.9-42.3) that likewise offers low dreariness and quick recuperation. Albeit no volumetric bosom estimations were acted in this arrangement, it very well may be derived from the mastectomy weight estimated for every patient (mean weight, $210 \mathrm{~g}$ : extend, 120-380 g) that a huge, variable bosom volume can be accomplished with the procedure.

This paper features the all-encompassing alternatives for fat uniting beneficiary. The pectoralis major and serratus muscles were not fat joined in quick bosom recreations following malignant growth evacuation, due to the worry that the infusion may disperse any leftover tumor tissues, prompting neighborhood metastases.

The idea and hypothesis of infusion metastasis stays disputable, and there is almost no clinical proof to help an absence of disease chance because of a bosom recreation. In vitro investigations have essentially insinuated an expanded danger of bosom recreation advancing malignancy or metastases, in spite of the fact that these outcomes have not been affirmed in vivo. We have not watched this relationship in our training, albeit long haul oncologic follow-up is required for both autologous and embed based bosom reconstructions [24].

The effect of performing concurrent fat joining with a LD fold move in a patient that has experienced radiation treatment, alongside deciding safe fat uniting volumes and fat endurance in this unique situation, is a territory that requires further research. The volume of fat united during a synchronous fat joining meeting and the quantity of meetings of ensuing fat uniting required were both multifactorial and individualized, affected by the patient's habitus, the size of the patient's LD muscle and LD skin paddle, the contralateral bosom volume, conceivable fat uniting beneficiary destinations, and the patient's desires for their reconstruction [24].

Patients with a huge LD muscle and LD skin paddle ordinarily have progressively fat uniting beneficiary tissues accessible. Reaping the biggest potential skin paddle and the whole LD muscle is basic for accomplishing the biggest wanted bosom volume. The structure of the skin oar can be made by surveying the benefactor site skin notch and playing out a squeeze test, which can assist with arranging the biggest skin paddle and keep up a very much covered giver site scar [23].

As of late, fat uniting has been demonstrated to be a promising remedial methodology for scar the executives after consumes and physical injury. Along these lines, there is proof to propose that early fat joining may help lessen scar tissue arrangement. This strategy can be utilized in the setting of a LD fold/tissue expander/embed recreation, and could likewise be extended to different autologous bosom remaking choices, including the transverse upper gracilis fold, the profunda course perforator fold, and the profound second rate epigastric perforator/musclesparing free transverse rectus abdominus myocutaneous fold bosom reconstruction [4].

This fundamental report portrays our initial encounters with using the fat-united, volume upgraded LD fold move and fat joining beneficiary destinations in simply autologous bosom remaking. The translation of our outcomes is restricted by this current examination's modest number of patients and short follow-up period for patients. Numerous meetings of fat uniting are as yet required now and again to accomplish the patient's ideal outcomes, contingent upon her last wanted volume for the reproduced bosom.

\section{Conclusion}

The LD fold move with synchronous fat uniting in prompt bosom remaking is a basic and safe method for autologous bosom reproduction.

Our experience has demonstrated that fat uniting can be performed securely and effectively alongside the autologous LD fold move utilizing a multisite, multilayer approach.

This methodology can possibly be utilized in tertiary bosom remaking too and can be effectively done in patients who have recently experienced radiation 
treatment, without an expanded danger of entanglements, including fat putrefaction.

The method offers an elective way to deal with autologous bosom reproduction that can give bigger bosom volumes without the requirement for forceful LD fold reaping, which is related with expanded giver site horribleness.

Using fat-united, volume upgraded LD fold move and fat joining beneficiary locales is a promising methodology for complete autologous bosom remaking in patients with restricted contributor site accessibility.

\section{Refereences}

[1] W.P. Audretsch, M. Rezai , Kolotas C, Tumorspecific immediate reconstruction (TSIR) in breast cancer patients. Perspect Plast Surg, Vol.11, PP.71100,1998..

[2] D.W. Chang, A. Youssef, S. Cha, G.P., Reece. "Autologous breast reconstruction with the extended latissimus dorsi flap." Plast Reconstr Surg. 2002:110:751-759.

[3] M. Economides, James , H. Song David, "Latissimus Dorsi and Immediate Fat Transfer (LIFT) for Complete Autologous Breast Reconstruction". Plast Reconstr Surg Glob Open, vol.6, PP.e1656, 2018.

[4] M. Alharbi, , I. Garrido, , C. Vaysse, Latissimus dorsi flap invasion by ductal breast carcinoma after lipofilling. Plastic and Reconstructive Surgery Global Open,Vol.1(8), PP.213, 2013

[5] H.R. Shin, K.W. Jung, Y.J. Won ,Annual report of the Korea Central Cancer Registry: based on registered data from 139 hospitals. Cancer Res Treat, Vol.36, PP.103-114,2004.

[6] M. Hamdi Oncoplastic and reconstructive surgery of the breast Breast, Vol.22 Suppl 2, PP.S1005,2013 .

[7] D.W. Chang, A. Youssef, S. Cha, “Autologous breast reconstruction with the extended latissimus dorsi flap." Plast Reconstr Surg, vol.110, PP.751$759,2002$.

[8] O.A. Branford, N. Kelemen, C.E. Hartmann, "Subfascial harvest of the extended latissimus dorsi myocutaneous flap in breast reconstruction: a comparative analysis of two techniques". Plast Reconstr Surg, vol.132, PP. 737-748,2013.

[9] Jeremy Niddam, Luciano Vidal, Barbara Hersant, "Primary Fat Grafting to the Pectoralis Muscle during Latissimus Dorsi Breast Reconstruction", Plast Reconstr Surg Glob Open, vol.4, PP.e1059, 2016.

[10] N.I.H. Conference,"Treatment of early-stage breast." JAMA,Vol.265 ,PP.391 - 5,1991.

[11] B. Fisher, S. Anderson, J. Bryant, Twenty-year follow-up of a randomized trial comparing total mastectomy, lumpectomy, and lumpectomy plus irradiation for the treatment of invasive breast cancer. New England Journal of Medicine, vol.347(16), PP. 1233-1241,2002.
[12] U. Veronesi, N. Cascinelli, L. Mariani, Twenty-year follow-up of a randomized study comparing breastconserving surgery with radical mastectomy for early breast cancer. New England Journal of Medicine, vol.347(16), PP.1227-1232,2002.

[13] L. R. Schover, Sexuality and body image in younger women with breast cancer. Journal of the National Cancer Institute. Monographs, vol.(16),PP. 177182,1994.

[14] P. Schrenk, Surgical and plastic reconstructive therapy of breast carcinoma. Wiener medizinische Wochenschrift, vol.150(4), PP.63-71,2000.

[15] J .C. Boughey, K. Hunt , E.K. Beahm "Onco plastic surgery for breast cancer." In Text book of surgical oncology., by R Daniel Beauchamp, Theo J M Ruers Graeme J Poston,vol.25(2), PP. 127-145,2007.

[16] G.F. Schwartz, U. Veronesi, K.B. Clough Consensus conference on breast conservation. ." J Am Coll Surg, vol.03, PP.198e207,2006.

[17] I. Tansini, Nuovo processo per amputations della mammella per cancro. Reform Med,Vol.12,PP. 35,1896 .

[18] W. M. Rozen, , A. K. Rajkomar, N. S. Anavekar Post-mastectomy breast reconstruction: a history in evolution, vol.25(2), PP.202-208 2009

[19] D. W. Chang, Y. Barnea, G. L. Robb, Effects of an autologous flap combined with an implant for breast reconstruction: an evaluation of 1000 consecutive reconstructions of previously irradiated breasts. Plastic and reconstructive surgery , Vol.122(2), PP.356-362,2008.

[20] A. M. Munhoz, E. Montag, E. G. Arruda, Management of giant inferior triangle lumbar hernia (Petit's triangle hernia): A rare complication following delayed breast reconstruction with extended latissimus dorsi myocutaneous flap. International journal of surgery case reports, vol.5(6), PP.319-323, 2014.

[21] D. ELFadl, V. Garimella, T. Mahapatra, (2010). Lipomodelling of the breast: a review. The Breast, vol.19(3),PP. 202-209, 2010.

[22] R. Sinna, E. Delay, S. Garson, Breast fat grafting (lipomodelling) after extended latissimus dorsi flap breast reconstruction: a preliminary report of 200 consecutive cases. Journal of plastic, reconstructive \& aesthetic surgery,Vol.63(11), PP.1769-1777,2010.

[23] Y. Jia, L. Zhu, A. Zeng, Latissimus dorsi flap for total autologous immediate breast reconstruction without implants. Plastic and reconstructive surgery,Vol.136(2),PP. 267e-268e, 2015.

[24] L. Zhu, A. T. Mohan, A. Vijayasekaran, Maximizing the volume of latissimus dorsi flap in autologous breast reconstruction with simultaneous multisite fat grafting. Aesthetic surgery J., Vol.36(2), PP.169-178,2016. 